\title{
CORRIGENDUM
}

\section{Frequent overexpression of ETS-related gene-1 (ERG1) in prostate cancer transcriptome}

G Petrovics, A Liu, S Shaheduzzaman, B Furusato, C Sun, Y Chen, M Nau, L Ravindranath, Y Chen, A Dobi, V Srikantan, IA Sesterhenn, DG McLeod, M Vahey, JW Moul and S Srivastava

Oncogene (2007) 26, 6684; doi:10.1038/sj.onc.1210745

Correction to:

Oncogene (2005) 24, 3847-3852. doi:10.1038/sj.onc.1208518; published online 7 March 2005
Since the publication of the above manuscript, the authors have identified an error in the author list; the name of the fourth author was misspelt. The corrected author list is shown above.

\section{Optimal primer design using the novel primer design program: MSPprimer provides accurate methylation analysis of the $A T M$ promoter}

\author{
JC Brandes, H Carraway and JG Herman
}

Oncogene (2007) 26, 6684; doi:10.1038/sj.onc.1210781

\section{Correction to:}

Oncogene (2007) 26, 6229-6237; doi:10.1038/sj.onc.1210433; published online 26 March 2007

Since the publication of the above paper, the authors found an error in two primer sequences in Table 1. The

correct primers should be ATM MSP2 M antisense CTCTaaCGaAAaCGATTaaCTaCCG and ATM MSP3 $M$ sense CGTTTGtTtCGACGGGtC. In the first case, the primer is missing an ' $a$ '. The second primer was inadvertantly a copy of the MSP3 M antisense primer. 